\title{
Many-Body Quantum Monte Carlo Study of 2D Materials: Cohesion and Band Gap in Single-Layer Phosphorene
}

\author{
T. Frank, ${ }^{1}$ R. Derian, ${ }^{2}$ K. Tokár, ${ }^{2}$ L. Mitas,${ }^{3}$ J. Fabian, ${ }^{1, *}$ and I. Štich ${ }^{2,4, \dagger}$ \\ ${ }^{1}$ Institute for Theoretical Physics, University of Regensburg, 93040 Regensburg, Germany \\ ${ }^{2}$ Center for Computational Materials Science, Institute of Physics, Slovak Academy of Sciences, \\ 84511 Bratislava, Slovakia \\ ${ }^{3}$ Department of Physics, North Carolina State University, Raleigh, North Carolina 27695-8202, USA \\ ${ }^{4}$ Institute of Informatics, Slovak Academy of Sciences, 84507 Bratislava, Slovakia \\ and Department of Natural Sciences, University of Saints Cyril and Methodius, 91701 Trnava, Slovakia
}

(Received 28 May 2018; revised manuscript received 19 November 2018; published 30 January 2019)

The quantum Monte Carlo (QMC) method is applied to obtain the fundamental (quasiparticle) electronic band gap $\Delta_{f}$ of a semiconducting two-dimensional phosphorene whose optical and electronic properties fill the void between graphene and 2D transition-metal dichalcogenides. Similarly to other 2D materials, the electronic structure of phosphorene is strongly influenced by reduced screening, making it challenging to obtain reliable predictions by single-particle density-functional methods. Advanced $G W$ techniques, which include many-body effects as perturbative corrections, are hardly consistent with each other, predicting the band gap of phosphorene with a spread of almost $1 \mathrm{eV}$, from 1.6 to $2.4 \mathrm{eV}$. Our QMC results, from infinite periodic superlattices as well as from finite clusters, predict $\Delta_{f}$ to be about $2.4 \mathrm{eV}$, indicating that available $G W$ results are systematically underestimating the gap. Using the recently uncovered universal scaling between the exciton binding energy and $\Delta_{f}$, we predict the optical gap of about $1.7 \mathrm{eV}$ that can be directly related to measurements even on encapsulated samples due to its robustness against dielectric environment. The QMC gaps are indeed consistent with recent experiments based on optical absorption and photoluminescence excitation spectroscopy. We also predict the cohesion of phosphorene to be only slightly smaller than that of the bulk crystal. Our investigations not only benchmark $G W$ methods and experiments, but also open the field of 2D electronic structure to computationally intensive but highly predictive QMC methods which include many-body effects such as electronic correlations and van der Waals interactions explicitly.

DOI: 10.1103/PhysRevX.9.011018

Subject Areas: Computational Physics, Condensed Matter Physics

\section{INTRODUCTION}

Two-dimensional materials have already revolutionized science, and have the potential to revolutionize technology due to their unique electronic, optical, thermal, spin, and magnetic properties [1-7]. Remarkably, 2D materials cover a wide range of electronic structures. The electronic properties range from metallic single atom layers of palladium and rhodium [8], semimetallic graphene [3], semiconducting transition-metal dichalcogenides [5], to insulating wide-gap hexagonal boron nitride $(h$-BN) [6]. Crucial for device applications are materials with a proper

\footnotetext{
*jaroslav.fabian@ur.de

ivan.stich@savba.sk
}

Published by the American Physical Society under the terms of the Creative Commons Attribution 4.0 International license. Further distribution of this work must maintain attribution to the author(s) and the published article's title, journal citation, and DOI. band gap. Layered black phosphorus (BP) features fundamental band gaps in the range of $0.3-2 \mathrm{eV}$, bridging semimetallic graphene and transition-metal dichalcogenides 9]]. This range is specifically important for optoelectronic, photovoltaic, photocatalytic, fiber optic telecommunications, and thermal imaging applications [10].

A single layer of black phosphorus-phosphorenecomprises $s p^{3}$ bonded phosphorus atoms forming an anisotropic puckered honeycomb lattice, see Fig. 1, which gives rise to special transport and spin relaxation properties in phosphorene [11,12]. The threefold bonding coordination implies that each phosphorus atom has a lone pair orbital which makes phosphorene reactive to air [9]. This oxidation degradation is eliminated by capping or encapsulating phosphorene with(in) an insulator.

Black phosphorus features a direct band gap at the $\Gamma$ point, all the way from single-layer phosphorene up to bulk. However, and this makes phosphorene particularly attractive, there are several ways to manipulate the gap. (i) First, the gap varies with the number of layers and therefore band 


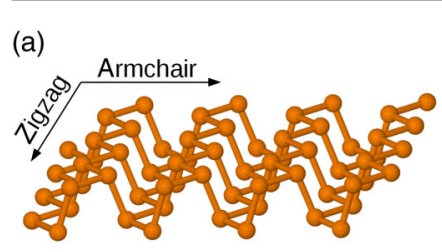

(c)

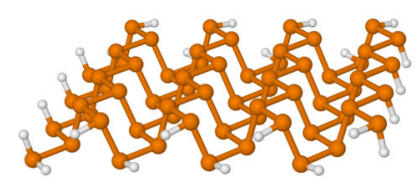

(b)

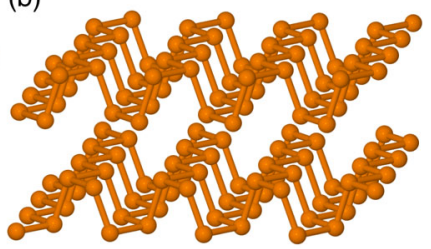

(d)

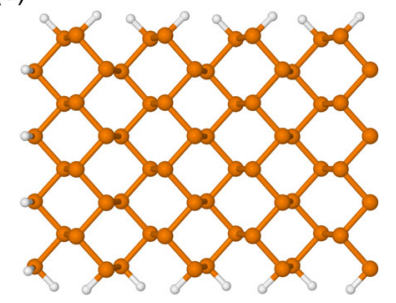

FIG. 1. Atomic structure of (a) single-layer and (b) few-layer phosphorene. Characteristic armchair and zigzag directions are indicated in (a). Side and top views of a $4 \times 4$ cluster approximant, with saturated edge bonds, are presented in (c) and (d), respectively.

engineering techniques can be readily applied. Exfoliation of layers from the bulk BP is straightforward [1,2,13,14], see Fig. 1, due to predominantly van der Waals interlayer bonds [15]. While in the bulk the gap is about $0.3 \mathrm{eV}$, it increases towards about $2 \mathrm{eV}$ (we argue for $2.4 \mathrm{eV}$ ) in single-layer phosphorene [10]. (ii) Next, phosphorene can sustain large in-plane compressive or tensile strains in excess of about $10 \%$, compared to just some $2 \%$ in the bulk [16]. This strain engineering is predicted to affect the band gap by $\approx \pm 50 \%[17,18]$. Finally, (iii) the gap of phosphorene is predicted to be strongly susceptible to the dielectric environment $[2,10,13]$.

Assuming that the fundamental (quasiparticle) band gap $\Delta_{f}$ depends on the dielectric that protects it against degradation, can we infer from experiments some key characteristics about pristine phosphorene providing thus the benchmark for both experiment and theory? Two established facts make the answer positive. (a) The dielectric environment affects both the fundamental gap as well as the exciton binding energy $\Delta_{b}$ [19]. Remarkably, the difference, $\Delta_{o}=\Delta_{f}-\Delta_{b}$, which is the optical gap, is essentially unaffected by the dielectric [20-25]. (b) Recently, a universal linear scaling between the exciton binding energy and the fundamental gap, $\Delta_{b} \approx 0.27 \Delta_{f}$, was predicted based on many examples from the 2D realm [26] (see also the predecessor [27]), including phosphorene. Combining these two observations, (a) and (b), allows us to estimate $\Delta_{o}$ from a calculation of $\Delta_{f}$ on a pristine $2 \mathrm{D}$ material, namely $\Delta_{o} \approx 0.73 \Delta_{f}$, and compare with experimental $\Delta_{o}$ obtained from an encapsulated or capped sample.

Let us now turn to the existing experimental and theoretical state of the art in determining the electronic gap(s) of single-layer phosphorene. Table I shows a rather comprehensive selection of measured and calculated

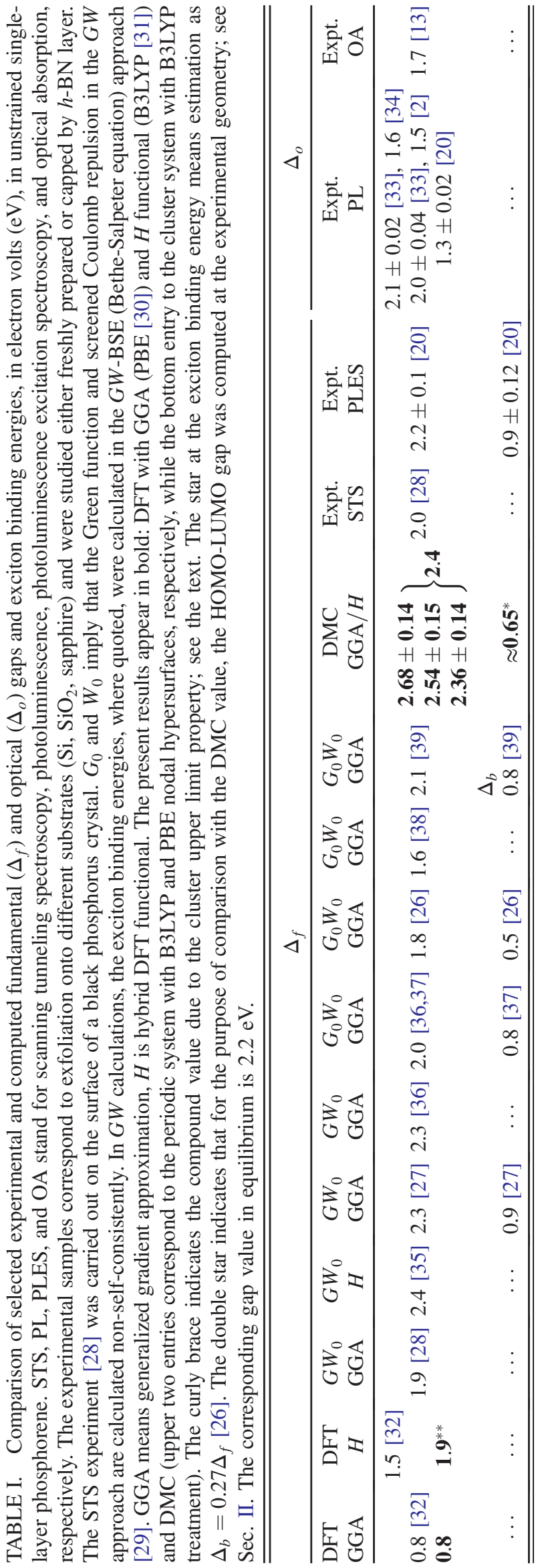


fundamental and optical gaps, as well as exciton binding energies for phosphorene. Photoluminescence is often contaminated by defect emission, as is also evidenced by the scatter of the measured values for $\Delta_{o}$. Most reliable is optical absorption. A recent experiment [13] has reported $\Delta_{o} \approx 1.73 \mathrm{eV}$, for phosphorene encapsulated in $h$-BN. This value would lead to $\Delta_{f} \approx 2.4 \mathrm{eV}$ for pristine phosphorene, according to the abovementioned linear scaling. Similarly, it would suggest the exciton binding energy $\Delta_{b}$ of about $0.65 \mathrm{eV}$. Certainly both (a) and (b) observations are not exact, so the above estimates of $\Delta_{f}$ and $\Delta_{b}$ would carry a scatter of perhaps $10 \%$ or so. If we next look at the photoluminescence excitation spectroscopy data for phosphorene on silicon oxide [20], the obtained fundamental gap is $\Delta_{f} \approx 2.2 \pm 0.1 \mathrm{eV}$. Considering the influence of the oxide, it is reasonable to deduce from this experiment that the fundamental gap of freestanding phosphorene is $0.1-0.2 \mathrm{eV}$ greater, that is, $2.3-2.4 \mathrm{eV}$ [20].

On the theory side, we see from Table I that single-particle density-functional methods predict, unsurprisingly, too low and strongly method-dependent values for $\Delta_{f}$. Inclusion of $G W$ corrections [29] is essential to bring the gaps closer to $2 \mathrm{eV}$. However, various $G W$ approximants $\left(G_{0} W_{0}\right.$ or $\left.G W_{0}\right)$ give different values coming from different implementations, ranging from 1.6 to $2.4 \mathrm{eV}$. The largest value, $2.4 \mathrm{eV}$, which would be consistent with the aforementioned optical absorption experiments, is obtained by using a hybrid functional [35]. However, the same implementation predicts $0.6 \mathrm{eV}$ band gap for bulk BP [35] (experimental value is $0.3 \mathrm{eV}$ ), making it clear that there is a limited predicting power from this calculation. As for the exciton binding energies, predictions (see Table I) range from 0.5 to $1 \mathrm{eV}$, again with little consensus in both theory and experiment. The experimental value of $0.9 \mathrm{eV}$ [20] is likely affected by the optical edge of $1.3 \mathrm{eV}$ of the emission peak (compare to $1.73 \mathrm{eV}$ of the absorption experiment [13]).

To obtain accurate bounds and reliable estimates of the band gap of phosphorene, we propose to employ the quantum Monte Carlo (QMC) method. The QMC method is an efficient, albeit computationally demanding [40,41], way to benchmark electronic structure calculations in condensed matter [42-44]. Indeed, this method has been applied to compute gaps in three-dimensional systems [42], clusters [45], and nanoparticles [46]. In the 2D realm it was already used to obtain reference binding energies of 2D bilayers $[15,47]$, which compares favorably with highly accurate quantum chemistry calculations [48], but thus far has not been systematically employed to obtain electronic structure parameters. Given the large parameter space for tuning the electronic structure of 2D systems, such as layer and strain engineering or dielectric embedding and the large scatter of results produced by experimental methods and customary computational techniques, the robust QMC method lends itself naturally for benchmarking purposes and validation of the different methods in the realm of 2D materials.
Here we report the first step in that effort: the QMC calculation of the fundamental band gap of unstrained freestanding single-layer phosphorene. We use both the periodic lattice as well as cluster approaches to demonstrate convergence and consistency. The accuracy of the groundstate properties is evidenced by calculating cohesion, which differs little from the bulk value. We stress that our QMC calculation is the full method, not relying on phenomenological interactions. In fact, we solve the Schrödinger equation for hundreds of electrons interacting mutually as well as with the lattice ions, to obtain the ground and excited states needed for the band gap calculation. Finally, we note that the knowledge of the band gap of phosphorene is important not only on its own right as a fundamental electronic quantity of a potentially technologically relevant material, but it is crucial also for building effective theories such as tight-binding [38] and $k \cdot p$ models [49]. We believe that our adaptation of QMC methods will open the way for this powerful technique to investigations of electronic structures of $2 \mathrm{D}$ systems, which are inherently prone to strong interactions and require careful considerations.

\section{SIMULATION TECHNIQUES}

The fundamental gap $\Delta_{f}$ was determined from both extended and cluster approximants with lattice parameters fixed to the experimental values in the black phosphorus crystal. In fact, it was shown that the experimental lattice parameters agree with the lattice parameters determined by QMC methods within the error bars [15]. The gap $\Delta_{f}$ was extracted as the singlet-singlet vertical excitation energy. Here $\Delta_{f} \approx E_{v}^{s s} \equiv E_{1}^{s}-E_{0}^{s}$, with $E_{0}$ and $E_{1}$ being, respectively, the ground and the first excited states obtained by the fixed-node QMC method [40] not allowing any relaxation of the density-functional theory (DFT) nodal hypersurfaces due to the HOMO $\rightarrow$ LUMO electron excitation; no vibronic effects are included. The fixed-node approximation is the only fundamental approximation in the electronic structure QMC method [40].

In the periodic setup, $E_{0}$ and $E_{1}$ were computed from DMC (diffusion Monte Carlo) energies in the fixed-node approximation using the variational Monte Carlo trial wave functions with the nodal hypersurfaces determined by two different sets of DFT orbitals: the generalized gradient approximation Perdew-Burke-Ernzerhof (PBE) [30] (DMC@PBE) and hybrid Becke three-parameter Lee-Yang-Parr (B3LYP) [31] (DMC@B3LYP), at the $\Gamma$ point of the Brillouin zone, optimizing the short-range correlations of the Jastrow factor [40]. The consistency check using both PBE and B3LYP DFT nodal hypersurfaces was deemed important, as at the DFT level the HOMO-LUMO gaps of the two DFT functionals differ by $\approx 1 \mathrm{eV}$; see Table I. The Yeh-Berkowitz [50] modification in the 3D Ewald summation technique for systems with a 
slab geometry that are periodic in two dimensions and have a finite length in the third dimension was adopted. We cross-checked in detail that this agrees with an alternative derivation of Ewald sums for 2D slab geometries [51]. In the cluster setup we used the B3LYP [31] (DMC@B3LYP) nodal hypersurfaces.

Finite-size scaling towards the thermodynamic limit was performed for a series of $1 \times 1$ to $6 \times 6$ series of $L \times L$ periodic approximants, see Fig. 1(a), and for $4 \times 4,5 \times 5$, and $6 \times 6 \mathrm{H}$-terminated cluster approximants, assuming a linear scaling with $1 / N$, see Supplemental Material (SM) [52] for more details, where $N=4 \times L \times L$ is the number of $\mathrm{P}$ atoms. Our approach corresponds to quasiexact manybody treatment, to within the fixed-node approximation, of the $2 \mathrm{D}$ electron polarizability entering the equation for $\Delta_{f} \propto 1 / \alpha_{2 \mathrm{D}}$. [26] The ground-state energy $E_{0}$ was also used to determine the cohesion energy. More details can be found in the SM [52].

\section{RESULTS AND DISCUSSION}

\section{A. Periodic supercells}

Our finite-size DMC scaling study of $\Delta_{f}$ is shown in Fig. 2(a). Although in 3D periodic calculations typical
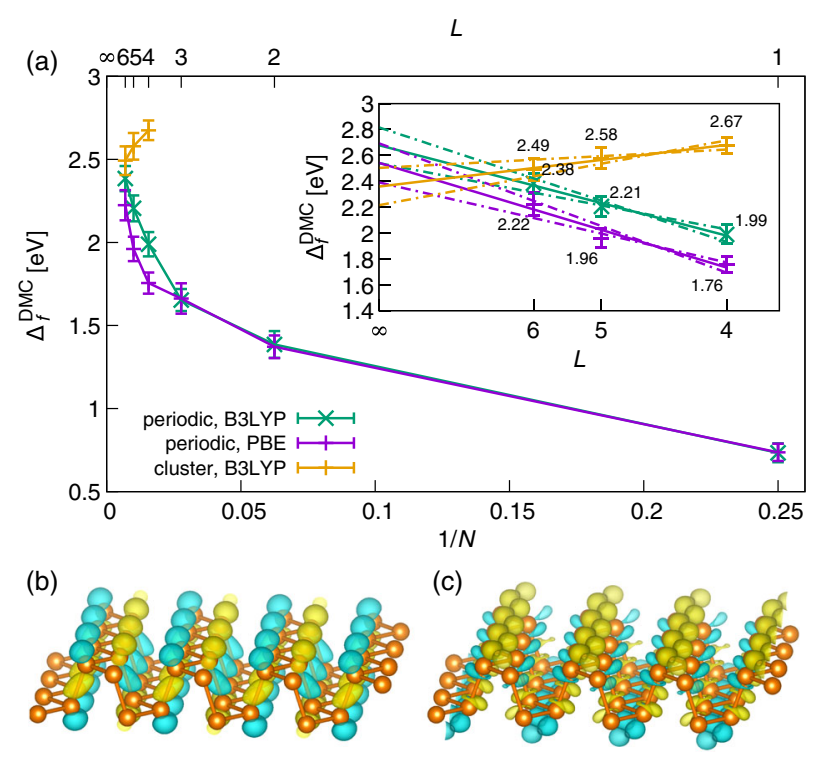

FIG. 2. (a) Finite-size scaling of the computed fundamental gap $\Delta_{f}$, with the number of atoms $N$ in the periodic supercell (green line, DMC@B3LYP; purple line, DMC@PBE) and in the cluster approach (yellow line, DMC@B3LYP) along the series of $L \times L$ supercell approximants, $L=1-6$ for the periodic setup and $L=$ 4-6 in the cluster approach. The inset shows an enlargement of the scaling for large $N$ with the dashed lines showing the linear extrapolation to the infinite-size limit. (b) B3LYP DFT HOMO (left) and (c) LUMO (right) orbitals. Both are $\Gamma$-point Bloch states, HOMO being a superposition of bonding orbitals [mostly $\sigma\left(p_{z}\right)$ ] along the vertical $\mathrm{P}-\mathrm{P}$ bonds while LUMO is its antibonding counterpart.
QMC extrapolations from supercells to bulk behave as $1 / N$, where $N$ is the number of atoms in the supercell, in the 2D slab systems the issue is more complicated [51]. The strong periodic Coulomb interactions unscreened in the direction perpendicular to the layer make the renormalization of electron interactions huge and the electrostatic energies slowly convergent. Therefore, the supercells have to be sufficiently large to marginalize the periodicity effects and to allow for reliable finite-size extrapolations. To this end, we have carried out extensive calculations of $\Delta_{f}$ for the sequence of $1 \times 1$ up to $6 \times 6$ periodic supercell systems with two DFT nodal hypersurfaces. For sizes $3 \times 3$ or larger, the linear scaling describes the results very well. For smaller supercells, the different components of the total energies, such as the potential energy, are too biased to make the trends transparent.

The infinite-size extrapolation from the extended-system calculations yields

$$
\Delta_{f}^{\mathrm{ext}, 1}=2.68 \pm 0.14 \mathrm{eV}
$$

fixing the nodal hypersurfaces by the hybrid B3LYP [31] DFT functional. Recall that the corresponding DFT value is $1.9 \mathrm{eV}$; see Table I. If we now fix the nodal hypersurfaces by PBE [30] DFT single-particle orbitals, see Fig. 2(a), we get the extrapolation to

$$
\Delta_{f}^{\mathrm{ext}, 2}=2.54 \pm 0.15 \mathrm{eV}
$$

which overlaps with $\Delta_{f}^{\text {ext,1 }}$ within the error bars. The PBE DFT gap is $0.8 \mathrm{eV}$. Remarkably, despite the significant difference of more than $1 \mathrm{eV}$ in the DFT gaps, the QMC method that starts with the corresponding DFT wave functions (and fixing their nodal hypersurfaces) gives a consistent output for both. Surprisingly, the convergence of $\Delta_{f}$ in the periodic setting is determined mainly by the Hartree-Fock energy components, with the rest, including the correlation energies, converging much faster. For example, the ground-state correlation energy is essentially converged at the $3 \times 3$ supercell size, whereas the excited state is only converged at a slightly larger size of $5 \times 5$. This provides a way of estimating the phosphorene properties from the more slowly converging Hartree-Fock results, simply by adding the corresponding correlation energies; see SM for more details [52]. We also plot the HOMOLUMO orbitals for the extended calculations at the $\Gamma$ point in Figs. 2(b) and 2(c), respectively. The orbital's distribution and bonding type can give insight into Coulomb finite size effects; see SM for a discussion [52].

\section{B. Clusters}

Because of intricacies of extrapolations of the periodic supercells, and for consistency, we have also calculated $\Delta_{f}$ in a finite cluster setting. The clusters corresponded to supercell sizes in the periodic method above, with 
terminations of unsaturated bonds with $\mathrm{H}$ atoms; see Figs. 1(c) and 1(d). Finite-size scaling constructed from these cluster approximants, given in Fig. 2(a), extrapolates to

$$
\Delta_{f}^{\text {clst }}=2.36 \pm 0.14 \mathrm{eV},
$$

reasonably close to $\Delta_{f}^{\text {ext }}$ considering the fact that we compare periodic systems versus isolated clusters in vacuum and that these two models actually exhibit opposite trends as the functions of the system size. Because of finite-size confinement, the computed excitations in finite clusters as a rule overestimate the gaps. The DMC values for the $5 \times 5$ and $6 \times 6$ clusters clearly illustrate this; see Fig. 2(a). However, as explained in SM, the cluster gaps are bound to converge to the fundamental gap in the infinite-size limit and also provide upper bounds for the estimation of fundamental gaps as indicated above [52]. The upper bound property of cluster gaps enables us to probe the usefulness of alternative extrapolations to bulk schemes, such as, for instance, $1 / \sqrt{N}$ scaling [71]; see SM [52]. Since both our estimates of $\Delta_{f}$ from the periodic supercells are greater than the cluster estimate, there is likely a small bias in the evaluation of excitations in the periodic supercells. In particular, the fixednode errors in the excited and the ground states are most likely not identical and could also be intertwined with the remnants of the finite-size errors even at larger sizes.

The convergence of the excited states is much more challenging in extended 2D systems than in bulk. The key reason is that the nonperiodic direction enables changes in the single-particle densities that have dominant contribution to the periodic Coulomb interactions, a problem that is naturally avoided in cluster geometries; for details see SM [52]. Therefore, we estimate that a systematic uncertainty of $0.1-0.2 \mathrm{eV}$ could be present in our periodic-supercells gap calculations. The multiple calculations of the excited states that we present clearly illustrate this aspect.

The error is certainly greater for the excited-state energy than for the ground state. Since the variational principle behind the QMC model gives the upper bound on the energy, it follows that the cluster value is a superior estimate for the fundamental gap. We believe that the true value of $\Delta_{f}$ for intrinsic phosphorene is about $2.4 \mathrm{eV}$, as also indicated in Table I. The $G W$ values, defining the current state of the art, are, compared to QMC values, widely scattered and systematically underestimating the gap.

\section{Exciton binding energy, optical gap, and comparison with experiment}

Using the universal linear scaling [26], $\Delta_{b} \approx 0.27 \Delta_{f}$, we can also estimate the excitonic binding energy of phosphorene as $\Delta_{b} \approx 0.65 \mathrm{eV}$. The optical gap is then $\Delta_{o}=\Delta_{f}-\Delta_{b} \approx 1.75 \mathrm{eV}$. This is consistent with the optical absorption experiment [13], which reports $1.73 \mathrm{eV}$. As we discussed in Sec. I, the optical gap of
2D semiconductors should be insensitive to the dielectric environment, justifying the consistency claim.

In terms of the fundamental gap obtained in experiment (see Table I), photoluminescence emission spectroscopy (silicon substrate, no capping) [20], optical absorption (sapphire substrate, $h$-BN capping) [13], and scanning tunneling spectroscopy (no capping) [28] yield values of $\Delta_{f}$ of $2.2,1.8$, and $2.0 \mathrm{eV}$, respectively. The optical absorption value was attributed to the $h$-BN capping, which also seems to reduce the exciton binding energy to just $0.1 \mathrm{eV}$ [13]. The photoluminescence value of $2.2 \mathrm{eV}$ [20] is closest to our QMC prediction. Considering that the sample was on a dielectric substrate which lowers $\Delta_{f}$ by perhaps $0.1-0.2 \mathrm{eV}$ [20], our result is also consistent with this experiment.

\section{Cohesion energy}

One important quantity which, to the best of our knowledge, has not been determined experimentally yet, is the freestanding phosphorene cohesion energy. The cohesion energy of bulk black phosphorus is $3.43 \mathrm{eV} /$ atom [72]. Compared to ordinary semiconducting 3D materials the phosphorus crystal is quite different being a van der Waals system of covalently bonded slabs. Even though the system shows moderately large cohesion, the stability of black phosphorus is rather low, mainly due to an easy detachment of $\mathrm{P}_{4}$ molecular units that per atom are bonded almost as strongly as the bulk material. Having calculated the phosphorene DMC ground-state energies for a variety of sizes, we can estimate the cohesion energy in the thermodynamic limit. Since the $\mathrm{P}_{4}$ molecule is the dominant sublimation product from phosphorus solids, to estimate the cohesion we follow the thermodynamic path $\mathrm{P}_{4}$ molecule $\rightarrow$ bulk black phosphorus $\rightarrow$ phosphorene using also the QMC calculation of the van der Waals binding energy estimated recently [15]. The finite-size scaling, Fig. 3, yields a

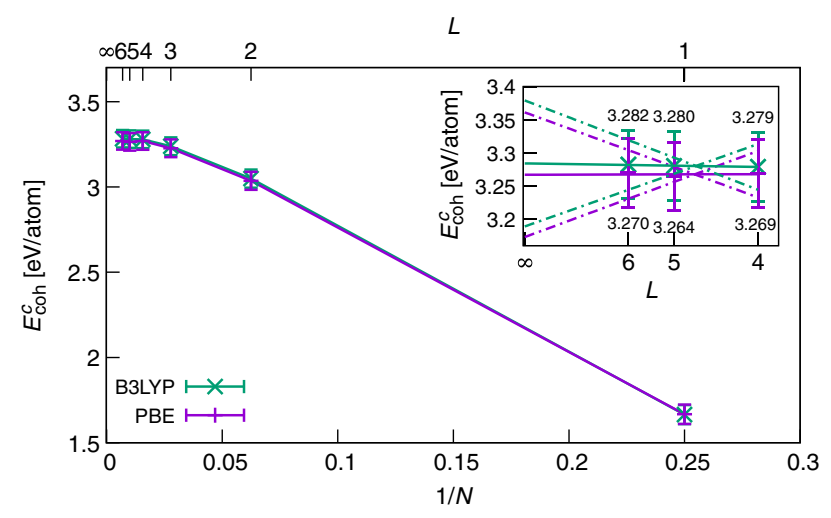

FIG. 3. Finite-size scaling of fixed-node corrected cohesion energy $E_{\text {coh }}^{c}$, in DMC@PBE (purple curve) and DMC@B3LYP (green curve) treatment. The error bars are smaller than the size of the points. The inset shows the enlargement of the linear scaling for large $N$ with extrapolation to infinite system size. 
fixed-node corrected cohesion energy of $3.27 \pm 0.1$ in DMC@PBE and 3.28 $\pm 0.06 \mathrm{eV} /$ atom in the DMC@B3LYP periodic calculations, while from the clusters the estimated value is $3.26 \pm 0.05 \mathrm{eV} /$ atom; see the $\mathrm{SM}$ for more details [52]. Note that our estimated DMC values of cohesion energy of phosphorene from the two models are almost identical and very close to the estimation of $3.35 \mathrm{eV} /$ atom for the $2 \mathrm{D}$ system obtained by using the van der Waals interlayer bonding from another DMC calculation [15] or coupled cluster result [48]. The corresponding DFT values are $3.09 \mathrm{eV} /$ atom [73] and $3.45 \mathrm{eV} /$ atom [74].

\section{CONCLUSION AND PERSPECTIVES}

We performed systematic fixed-node QMC calculations of the quasiparticle band gap of freestanding single-layer phosphorene in both periodic and cluster settings. Using the universal linear scaling between the gap and the optical binding energy, we also extracted the optical gap, which can be compared with experiments done on phosphorene on dielectric substrates or on encapsulated samples. Our results are consistent with available optical absorption and photoluminescence emission spectroscopy experiments. We argue that previous calculations based on $G W$ underestimate the quasiparticle gap and do not give consistent predictions for phosphorene. Our ground state is very accurate and limited only by the (corrected) fixed-node errors, evidenced from the calculated cohesion and its agreement with available (indirect) experimental data.

Our calculated quantities, band gap and cohesion, are key inputs into more qualitative and approximate theories, such as tight binding and $k \cdot p$, as well as into experimental interpretations. In particular, there is a clear path of how our results can be modified to accommodate dielectric environments which enter the experiments, leaving the core of our QMC results unchanged. This can be done by BetheSalpeter modeling or by use of model dielectric screening [23] to find the appropriate value of the exciton binding energy. Hence, our explicitly many-body QMC results provide a reference ground for further studies on phosphorene based on strain and layer engineering as well as chemical doping and structural defects and indeed in any other 2D material. We also demonstrated that these cuttingedge calculations are now feasible for a range of $2 \mathrm{D}$ systems and we expect the QMC methods to find a place at the top of the list of the toolkit for studying 2D systems. This is underscored by the fact that the scatter of predicted values for electronic parameters is significant not only in phosphorene, but in other 2D materials as well $[23,25,75]$.

\section{ACKNOWLEDGMENTS}

R. D., K. T., and I. S. were supported by Agentúra pre Podporu Výskumu a Vývoja (Slovak Research and Development Agency) APVV-15-0759, VEGA-2/0162/ 15, and VEGA-2/0123/18 projects. T.F. was supported by Graduiertenkolleg Grant No. 1570, and the International Doctorate Program Topological Insulators of the Elite Network of Bavaria. J. F. acknowledges funding from the European Union's Horizon 2020 research and innovation program under Grant Agreement No. 696656, and DFG SFB 1277 (B07). T.F. and J.F. acknowledge the Gauss Centre for Supercomputing for funding this project by providing computing time on the GCS Supercomputer SuperMUC at Leibniz Supercomputing Centre. Contributions by L. M. have been supported by the NSF Grant No. DMR-1410639 and by the NSF Extreme Science and Engineering Discovery Environment (XSEDE) computer time allocation at the Texas Advanced Computing Center (TACC) that we acknowledge for providing HPC resources that contributed to the reported research results. R. D., K. T., and I. S. acknowledge that the results of this research have been obtained using the DECI resources Beskow at the PDC Center for High Performance Computing at the KTH Royal Institute of Technology, Stockholm, Sweden from the PRACE project NaM2D and Sisu based in Finland at CSC IT Center for Science with support from the PRACE DoCSiNaP project. We also gratefully acknowledge use of the Hitachi SR16000/M1 supercomputer system at CCMS/IMR, Tohoku University, Japan. We also acknowledge useful discussions with Martin Gmitra and Alexey Chernikov.

[1] L. Li, Y. Yu, G. J. Ye, Q. Ge, X. Ou, H. Wu, D. Feng, X. H. Chen, and Y. Zhang, Black Phosphorus Field-Effect Transistors, Nat. Nanotechnol. 9, 372 (2014).

[2] H. Liu, A. T. Neal, Z. Zhu, Z. Luo, X. Xu, D. Tománek, and P. D. Ye, Phosphorene: An Unexplored 2D Semiconductor with a High Hole Mobility, ACS Nano 8, 4033 (2014).

[3] K. S. Novoselov, Electric Field Effect in Atomically Thin Carbon Films, Science 306, 666 (2004).

[4] B. Hunt, J. D. Sanchez-Yamagishi, A. F. Young, M. Yankowitz, B. J. LeRoy, K. Watanabe, T. Taniguchi, P. Moon, M. Koshino, P. Jarillo-Herrero, and R. C. Ashoori, Massive Dirac Fermions and Hofstadter Butterfly in a van der Waals Heterostructure, Science 340, 1427 (2013).

[5] K. F. Mak, C. Lee, J. Hone, J. Shan, and T. F. Heinz, Atomically Thin $\mathrm{MoS}_{2}$ : A New Direct-Gap Semiconductor, Phys. Rev. Lett. 105, 136805 (2010).

[6] K. Watanabe, T. Taniguchi, and K. Hisao, Direct-Bandgap Properties and Evidence for Ultraviolet Lasing of Hexagonal Boron Nitride Single Crystal, Nat. Mater. 3, 404 (2004).

[7] W. Han, R. K. Kawakami, M. Gmitra, and J. Fabian, Graphene Spintronics, Nat. Nanotechnol. 9, 794 (2014).

[8] H. Duan, N. Yan, R. Yu, C.-R. Chang, G. Zhou, H.-S. $\mathrm{Hu}$, H. Rong, Z. Niu, J. Mao, H. Asakura, T. Tanaka, P. J. Dyson, J. Li, and Y. Li, Ultrathin Rhodium Nanosheets, Nat. Commun. 5, 3093 (2014).

[9] X. Ling, H. Wang, S. Huang, F. Xia, and M. S. Dresselhaus, The Renaissance of Black Phosphorus, Proc. Natl. Acad. Sci. U.S.A. 112, 4523 (2015). 
[10] A. Castellanos-Gomez, Black Phosphorus: Narrow Gap, Wide Applications, J. Phys. Chem. Lett. 6, 4280 (2015).

[11] A. Avsar, J. Y. Tan, M. Kurpas, M. Gmitra, K. Watanabe, T. Taniguchi, J. Fabian, and B. Özyilmaz, Gate-Tunable Black Phosphorus Spin Valve with Nanosecond Spin Lifetimes, Nat. Phys. 13, 888 (2017).

[12] M. Kurpas, M. Gmitra, and J. Fabian, Spin-Orbit Coupling and Spin Relaxation in Phosphorene: Intrinsic versus Extrinsic Effects, Phys. Rev. B 94, 155423 (2016).

[13] L. Li, J. Kim, C. Jin, G. Ye, D. Y. Qiu, F. H. da Jornada, Z. Shi, L. Chen, Z. Zhang, F. Yang, K. Watanabe, T. Taniguchi, W. Ren, S. G. Louie, X. H. Chen, Y. Zhang, and F. Wang, Direct Observation of the Layer-Dependent Electronic Structure in Phosphorene, Nat. Nanotechnol. 12, 21 (2016).

[14] F. Xia, H. Wang, and Y. Jia, Rediscovering Black Phosphorus as an Anisotropic Layered Material for Optoelectronics and Electronics, Nat. Commun. 5, 4458 (2014).

[15] L. Shulenburger, A. D. Baczewski, Z. Zhu, J. Guan, and D. Tománek, The Nature of the Interlayer Interaction in Bulk and Few-Layer Phosphorus, Nano Lett. 15, 8170 (2015).

[16] R. Roldán, A. Castellanos-Gomez, E. Cappelluti, and F. Guinea, Strain Engineering in Semiconducting TwoDimensional Crystals, J. Phys. Condens. Matter 27, 313201 (2015).

[17] K. Tokár, R. Derian, J. Brndiar, and I. Štich, Strain Control of Vibrational Properties of Few Layer Phosphorene, J. Appl. Phys. 120, 194305 (2016).

[18] C. Wang, Q. Xia, Y. Nie, and G. Guo, Strain-Induced Gap Transition and Anisotropic Dirac-like Cones in Monolayer and Bilayer Phosphorene, J. Appl. Phys. 117, 124302 (2015).

[19] J. Xiao, M. Zhao, Y. Wang, and X. Zhang, Excitons in Atomically Thin 2D Semiconductors and Their Applications, Nanophotonics 6, 1309 (2017).

[20] X. Wang, A. M. Jones, K. L. Seyler, V. Tran, Y. Jia, H. Zhao, H. Wang, L. Yang, X. Xu, and F. Xia, Highly Anisotropic and Robust Excitons in Monolayer Black Phosphorus, Nat. Nanotechnol. 10, 517 (2015).

[21] S. Latini, T. Olsen, and K. S. Thygesen, Excitons in van der Waals Heterostructures: The Important Role of Dielectric Screening, Phys. Rev. B 92, 245123 (2015).

[22] A. V. Stier, N.P. Wilson, G. Clark, X. Xu, and S. A. Crooker, Probing the Influence of Dielectric Environment on Excitons in Monolayer $\mathrm{WSe}_{2}$ : Insight from High Magnetic Fields, Nano Lett. 16, 7054 (2016).

[23] M. L. Trolle, T. G. Pedersen, and V. Véniard, Model Dielectric Function for 2D Semiconductors Including Substrate Screening, Sci. Rep. 7, 39844 (2017).

[24] A. Raja, A. Chaves, J. Yu, G. Arefe, H. M. Hill, A. F. Rigosi, T. C. Berkelbach, P. Nagler, C. Schüller, T. Korn, C. Nuckolls, J. Hone, L. E. Brus, T. F. Heinz, D. R. Reichman, and A. Chernikov, Coulomb Engineering of the Bandgap and Excitons in Two-Dimensional Materials, Nat. Commun. 8, 15251 (2017).

[25] Y. Cho and T. C. Berkelbach, Environmentally Sensitive Theory of Electronic and Optical Transitions in Atomically Thin Semiconductors, Phys. Rev. B 97, 041409(R) (2018).

[26] Z. Jiang, Z. Liu, Y. Li, and W. Duan, Scaling Universality between Band Gap and Exciton Binding Energy of
Two-Dimensional Semiconductors, Phys. Rev. Lett. 118, 266401 (2017).

[27] J.-H. Choi, P. Cui, H. Lan, and Z. Zhang, Linear Scaling of the Exciton Binding Energy versus the Band Gap of Two-Dimensional Materials, Phys. Rev. Lett. 115, 066403 (2015).

[28] L. Liang, J. Wang, W. Lin, B. G. Sumpter, V. Meunier, and M. Pan, Electronic Bandgap and Edge Reconstruction in Phosphorene Materials, Nano Lett. 14, 6400 (2014).

[29] P. Cudazzo, L. Sponza, C. Giorgetti, L. Reining, F. Sottile, and M. Gatti, Exciton Band Structure in Two-Dimensional Materials, Phys. Rev. Lett. 116, 066803 (2016).

[30] J. P. Perdew, K. Burke, and M. Ernzerhof, Generalized Gradient Approximation Made Simple, Phys. Rev. Lett. 77, 3865 (1996).

[31] P. J. Stephens, F. J. Devlin, C. F. Chabalowski, and M. J. Frisch, Ab Initio Calculation of Vibrational Absorption and Circular Dichroism Spectra Using Density Functional Force Fields, J. Phys. Chem. 98, 11623 (1994).

[32] Y. Cai, G. Zhang, and Y.-W. Zhang, Layer-Dependent Band Alignment and Work Function of Few-Layer Phosphorene, Sci. Rep. 4, 6677 (2015).

[33] A. Surrente, A. A. Mitioglu, K. Galkowski, W. Tabis, D. K. Maude, and P. Plochocka, Excitons in Atomically Thin Black Phosphorus, Phys. Rev. B 93, 121405 (2016).

[34] G. Zhang, S. Huang, A. Chaves, C. Song, V. O. Özçelik, T. Low, and H. Yan, Infrared Fingerprints of Few-Layer Black Phosphorus, Nat. Commun. 8, 14071 (2017).

[35] V. Wang, Y. Kawazoe, and W. T. Geng, Native Point Defects in Few-Layer Phosphorene, Phys. Rev. B 91, 045433 (2015).

[36] F. A. Rasmussen, P. S. Schmidt, K. T. Winther, and K. S. Thygesen, Efficient Many-Body Calculations for TwoDimensional Materials Using Exact Limits for the Screened Potential: Band Gaps of $\mathrm{MoS}_{2}$ h-BN, and Phosphorene, Phys. Rev. B 94, 155406 (2016).

[37] V. Tran, R. Soklaski, Y. Liang, and L. Yang, LayerControlled Band Gap and Anisotropic Excitons in FewLayer Black Phosphorus, Phys. Rev. B 89, 235319 (2014).

[38] A. N. Rudenko and M. I. Katsnelson, Quasiparticle Band Structure and Tight-Binding Model for Single- and Bilayer Black Phosphorus, Phys. Rev. B 89, 201408 (2014).

[39] F. Ferreira and R. M. Ribeiro, Improvements in the GW and Bethe-Salpeter-Equation Calculations on Phosphorene, Phys. Rev. B 96, 115431 (2017).

[40] W. M. C. Foulkes, L. Mitas, R. J. Needs, and G. Rajagopal, Quantum Monte Carlo Simulations of Solids, Rev. Mod. Phys. 73, 33 (2001).

[41] R. J. Needs, M. D. Towler, N. D. Drummond, and P. L. Ríos, Continuum Variational and Diffusion Quantum Monte Carlo Calculations, J. Phys. Condens. Matter 22, 023201 (2010).

[42] J. Kolorenč and L. Mitas, Applications of Quantum Monte Carlo Methods in Condensed Systems, Rep. Prog. Phys. 74, 026502 (2011).

[43] L. K. Wagner and D. M. Ceperley, Discovering Correlated Fermions Using Quantum Monte Carlo, Rep. Prog. Phys. 79, 094501 (2016). 
[44] M. Dubecký, L. Mitas, and P. Jurečka, Noncovalent Interactions by Quantum Monte Carlo, Chem. Rev. 116, 5188 (2016).

[45] L. Horváthová, M. Dubecký, L. Mitas, and I. Štich, Spin Multiplicity and Symmetry Breaking in Vanadium-Benzene Complexes, Phys. Rev. Lett. 109, 053001 (2012).

[46] R. Derian, K. Tokár, B. Somogyi, Á. Gali, and I. Štich, Optical Gaps in Pristine and Heavily Doped Silicon Nanocrystals: DFT versus Quantum Monte Carlo Benchmarks, J. Chem. Theory Comput. 13, 6061 (2017).

[47] E. Mostaani, N. D. Drummond, and V. I. Fal'ko, Quantum Monte Carlo Calculation of the Binding Energy of Bilayer Graphene, Phys. Rev. Lett. 115, 115501 (2015).

[48] M. Schütz, L. Maschio, A. J. Karttunen, and D. Usvyat, Exfoliation Energy of Black Phosphorus Revisited: A Coupled Cluster Benchmark, J. Phys. Chem. Lett. 8, 1290 (2017).

[49] P. Li and I. Appelbaum, Electrons and Holes in Phosphorene, Phys. Rev. B 90, 115439 (2014).

[50] I.-C. Yeh and M. L. Berkowitz, Ewald Summation for Systems with Slab Geometry, J. Chem. Phys. 111, 3155 (1999).

[51] B. Wood, W. M. C. Foulkes, M. D. Towler, and N. D. Drummond, Coulomb Finite-Size Effects in Quasi-TwoDimensional Systems, J. Phys. Condens. Matter 16, 891 (2004).

[52] See Supplemental Material at http://link.aps.org/ supplemental/10.1103/PhysRevX.9.011018, which includes Refs. [15,30,31,40,50,53-71], for the simulation details and data, estimation of the cohesion energy from experiments, a discussion of the finite-size scaling of the fundamental gap, as well as data for the Hartree-Fock fundamental gaps.

[53] J. P. Perdew, K. Burke, and M. Ernzerhof, Erratum: Generalized Gradient Approximation Made Simple, Phys. Rev. Lett. 78, E1396 (1997).

[54] A. Brown and S. Rundqvist, Refinement of the Crystal Structure of Black Phosphorus, Acta Crystallogr. 19, 684 (1965).

[55] L. Cartz, S. R. Srinivasa, R. J. Riedner, J. D. Jorgensen, and T. G. Worlton, Effect of Pressure on Bonding in Black Phosphorus, J. Chem. Phys. 71, 1718 (1979).

[56] B. Kiraly, N. Hauptmann, A. N. Rudenko, M. I. Katsnelson, and A. A. Khajetoorians, Probing Single Vacancies in Black Phosphorus at the Atomic Level, Nano Lett. 17, 3607 (2017).

[57] T. Yanai, D. P. Tew, and N. C. Handy, A New Hybrid Exchange Correlation Functional Using the CoulombAttenuating Method (CAM - B3LYP), Chem. Phys. Lett. 393, 51 (2004).

[58] L. K. Wagner, M. Bajdich, and L. Mitas, QWalk: A Quantum Monte Carlo Program for Electronic Structure, J. Comput. Phys. 228, 3390 (2009).

[59] R. Dovesi, R. Orlando, A. Erba, C. M. Zicovich-Wilson, B. Civalleri, S. Casassa, L. Maschio, M. Ferrabone, M. De La Pierre, P. D'Arco, Y. Noël, M. Causà, M. Rérat, and B. Kirtman, CRYSTAL14: A Program for the Ab Initio
Investigation of Crystalline Solids, Int. J. Quantum Chem. 114, 1287 (2014).

[60] M. Bajdich and L. Mitas, Electronic Structure Quantum Monte Carlo, Acta Phys. Slovaca. Rev. Tutorials 59, 81 (2009).

[61] K. E. Schmidt and J. W. Moskowitz, Correlated Monte Carlo Wave Functions for the Atoms He through Ne, J. Chem. Phys. 93, 4172 (1990).

[62] C. J. Umrigar and C. Filippi, Energy and Variance Optimization of Many-Body Wave Functions, Phys. Rev. Lett. 94, 150201 (2005).

[63] M. Casula, Beyond the Locality Approximation in the Standard Diffusion Monte Carlo Method, Phys. Rev. B 74, 161102 (2006).

[64] K. G. Reeves, Y. Yao, and Y. Kanai, Diffusion Quantum Monte Carlo Study of Martensitic Phase Transition Energetics: The Case of Phosphorene, J. Chem. Phys. 145, 124705 (2016).

[65] A. Karton and J. M. L. Martin, W4 Thermochemistry of $\mathrm{P}_{2}$ and $\mathrm{P}_{4}$. Is the CODATA Heat of Formation of the Phosphorus Atom Correct?, Mol. Phys. 105, 2499 (2007).

[66] D. Feller and K.A. Peterson, Re-examination of Atomization Energies for the Gaussian-2 Set of Molecules, J. Chem. Phys. 110, 8384 (1999).

[67] P. A. G. O'hare, B. M. Lewis, and I. Shirotani, Thermodynamic Stability of Orthorhombic Black Phosphorus, Thermochim. Acta 129, 57 (1988).

[68] P. A. G. O'Hare and W. N. Hubbard, Fluorine Bomb Calorimetry. Part 18. Standard Enthalpy of Formation of Phosphorus Pen Afluoride and Enthalpies of Transition between Various Forms of Phosphorus. Thermodynamic Functions of Phosphorus Pentafluoride between 0 and 1500 K, Trans. Faraday Soc. 62, 2709 (1966).

[69] M. Burkatzki, C. Filippi, and M. Dolg, Energy-Consistent Pseudopotentials for Quantum Monte Carlo Calculations, J. Chem. Phys. 126, 234105 (2007).

[70] J. P. Perdew, W. Yang, K. Burke, Z. Yang, E. K. U. Gross, M. Scheffler, G. E. Scuseria, T. M. Henderson, I. Y. Zhang, A. Ruzsinszky, H. Peng, J. Sun, E. Trushin, and A. Görling, Understanding Band Gaps of Solids in Generalized Kohn-Sham Theory, Proc. Natl. Acad. Sci. U.S.A. 114, 2801 (2017).

[71] N. D. Drummond, V. Zólyomi, and V. I. Fal'ko, Electronic structure of two-dimensional crystals of hexagonal boron nitride, TTI, QMC in the Apuan Alps VIII, Vallico Sotto, Tuscany, Italy, 2013, http://www.tcm.phy.cam.ac.uk/ $\sim$ mdt26/tti_talks/qmcitaa_13/drummond_tti2013.pdf.

[72] C. Kittel, Introduction to Solid State Physics, 8th ed. (John Wiley \& Sons, Inc., New York, 2005), p. 50.

[73] P. Ballone and R. O. Jones, Density Functional Study of Phosphorus and Arsenic Clusters Using Local and Nonlocal Energy Functionals, J. Chem. Phys. 100, 4941 (1994).

[74] P.-J. Chen and H.-T. Jeng, High Applicability of TwoDimensional Phosphorous in Kagome Lattice Predicted from First-Principles Calculations, Sci. Rep. 6, 23151 (2016).

[75] J. Ryou, Y.-S. Kim, K. C. Santosh, and K. Cho, Monolayer $\mathrm{MoS}_{2}$ Bandgap Modulation by Dielectric Environments and Tunable Bandgap Transistors, Sci. Rep. 6, 29184 (2016). 